\title{
Fidelity of Runx2 Activity in Breast Cancer Cells Is Required for the Generation of Metastases-Associated Osteolytic Disease
}

\author{
George L. Barnes, ${ }^{1}$ Kerri E. Hebert, ${ }^{1}$ Mohammad Kamal, ${ }^{1}$ Amjad Javed, ${ }^{2}$ Thomas A. Einhorn, ${ }^{1}$ Jane B. Lian, ${ }^{2}$ \\ Gary S. Stein, ${ }^{2}$ and Louis C. Gerstenfeld ${ }^{1}$
}

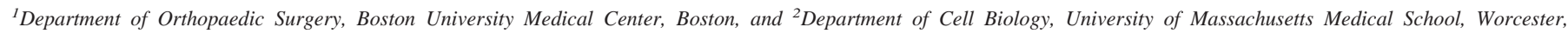
Massachusetts

\begin{abstract}
The osteolytic bone destruction associated with breast cancer skeletal metastases represents a serious and incurable clinical condition. However, the molecular mechanisms regulating tumor cell expression of factors involved in the generation of osteolytic disease remain elusive. We demonstrated recently that breast cancer cells express the Runx2 transcription factor, essential for bone formation and a regulator of skeletal homeostasis. Our experimental results demonstrate that perturbation of Runx2 regulatory function in tumor cells abolishes their ability to form osteolytic lesions in vivo. In vitro, we show that breast cancer cells inhibit osteoblast differentiation while concurrently enhancing osteoclast differentiation in marrow stromal cell cultures. Disruption of Runx 2 activity abrogates both of these cancer cell-mediated effects on bone cells. These results demonstrate that Runx2 expression in breast cancer cells provides a molecular phenotype that enables the interactions between tumor cells and the bone microenvironment that lead to osteolytic disease.
\end{abstract}

\section{INTRODUCTION}

It is widely recognized that breast cancer cells preferentially invade and grow as secondary tumors in bone. In 1889, Stephen Paget (1) proposed the "seed and soil" theory to explain the preferential metastasis of cancers to distant sites. Paget proposed that metastatic cancers, including breast, prostate, and lung, preferentially localize to bone, because they find bone to be a fertile soil on which to grow. The consequence of the successful seeding and growth of breast cancer cells in the bone environment is bone destruction; however, studies demonstrate that cancer cells do not directly erode bone. Instead, most of the induced bone destruction is mediated by the local osteoclasts $(2,3)$. Histologically, lytic tumors appear adjacent to active osteoclasts on the bone surface being resorbed, and inhibitors of osteoclastic activity, including bisphosphonates, have been successfully used in women with metastases to reduce the lytic loss of bone $(4,5)$.

There is debate currently over the primary mechanism by which breast carcinoma influence osteoclast formation and activity leading to osteolytic lesions. One side of the debate favors a direct influence of breast cancer cells on osteoclast formation. Breast cancer cells have been demonstrated to express several factors known to directly modulate osteoclast formation and activity, including macrophage colony stimulating factor, interleukin (IL) 6, IL-11, and IL-8 (6-10). Studies have experimentally shown the direct induction of osteoclast formation by these tumor-produced factors in vitro. IL-8, in particular, has been shown recently to directly induce osteoclast formation in peripheral blood monocytes (7). Interestingly, these studies additionally demonstrate that IL-8

Received 12/9/03; revised 3/29/04; accepted 4/30/04.

Grant support: NIH Grants AR 47045 (L. Gerstenfeld), AR 48818 (G. Stein), and CA 82834 (G. Stein). Institutional support was provided by the Department of Orthopaedic Surgery, Boston University School of Medicine (G. Barnes).

The costs of publication of this article were defrayed in part by the payment of page charges. This article must therefore be hereby marked advertisement in accordance with 18 U.S.C. Section 1734 solely to indicate this fact.

Requests for reprints: George L. Barnes, Department of Orthopaedic Surgery, Boston University Medical Center, 715 Albany Street, R-205, Boston, MA 02118. Phone: (617) 414-1664; Fax: (617) 414-1661; E-mail: gbarnes@bu.edu. also acts on osteoblasts inducing increased expression of the osteoclastogenic cytokine, the receptor activator of nuclear factor $\kappa \mathrm{B}$ ligand (RANKL). RANKL has been demonstrated to be one of the major inducers of osteoclast formation in bone. Thus, IL-8 appears to have direct and indirect effects on osteoclast formation. A number of other studies also support an indirect mechanism of tumor-induced osteoclast formation by parathyroid hormonerelated peptide. As with IL-8, parathyroid hormone-related peptide is expressed in many breast cancers, and parathyroid hormonerelated peptide has been reported to induce increased expression of RANKL in osteoblasts $(8,11-14)$. These studies also demonstrate that neither model breast cancer cell lines (MDA-MB-231, MCF-7, or T47D) nor primary tumors express the primary inductive factor in osteoclast formation, RANKL (11). Thus, data exists supporting both direct tumor-induced osteoclast formation and the indirect induction of osteoclasts by tumor cells through an osteoblastmediated effect. Neither model is mutually exclusive of the other, and it is likely that tumor cells induce osteoclast formation through a combination of these two mechanisms.

The term osteomimicry has been coined to describe the expression of bone-related genes in metastatic cancers that preferentially localize to skeletal sites. The expression of osteoblast-related genes by tumor cells is thought to play an important role in facilitating the interactions between cancer cells and the bone environment. Breast, prostate, and lung cancers have been demonstrated to express several molecules usually restricted to skeletal cells, including bone sialoprotein (BSP; Refs. 15-19). Our laboratory and others have demonstrated recently that metastatic breast cancer cells express the transcription factor Runx2 [also referred to as core binding factor $\alpha 1$ /acute myeloid leukemia (AML)-3], and that this transcription factor regulates the expression of skeletalrestricted genes in cancer cells (17-19). Runx2 is one of the primary transcriptional regulators of new bone formation and skeletal maintenance (20-24). Ablation of this gene in mice results in a complete absence of bone formation that is attributed to the arrest of chondrogenic and osteogenic maturation $(23,24)$. During osteogenesis, Runx 2 is expressed in both dividing osteoprogenitors and mature osteoblasts and plays a regulatory role in cellular proliferation and differentiation (25). Runx2 is involved in the expression of soluble factors that mediate angiogenic events (vascular endothelial growth factor), tissue remodeling (matrix metalloproteinases 9 and 13), and the coupling of osteoblasts to osteoclasts (e.g., osteoprotogerin/RANKL; Refs. 26-31). In mature osteoblasts, Runx2 transcriptionally regulates many of the genes associated with mineralization, including osteocalcin, BSP, and collagen type I $(23,24,32,33)$. Thus, this single transcription factor plays a functional role in multiple stage-specific functions of the osteoblast lineage. By extension, Runx 2 may regulate multiple factors in metastatic breast cancer cells involved in tumor-bone cell interactions.

Runx family members primarily act as scaffolds or molecular organizers, interacting with coregulatory factors, promoter elements, and specific subnuclear targets in the nuclear matrix (34). 
Functional interactions have been demonstrated between the Runx proteins and coregulators, including Groucho/transducin-like enhancer of split (TLE), retinoblastoma protein $(\mathrm{Rb}) \mathrm{CAAT} / \mathrm{enhancer}$ binding protein, yes-associated protein, and the Smads (34-40). Consensus binding elements for Runx 2 have been identified in multiple genes associated with skeletal homeostasis, angiogenesis, and mineralization $(23,24,32,33)$. Additionally, appropriate Runx regulatory function requires proper subnuclear targeting of Runx family members $(41,42)$. A clear example for this requirement is derived from the Runx1-ETO (AML1-ETO) fusion protein that is associated with acute myelogenous leukemia. This gene rearrangement $\mathrm{t}(8 ; 21)$ fuses the $\mathrm{NH}_{2}$-terminal portion of AML1 (Runx1) to the ETO (MTG8) repressor protein $(43,44)$. AML1-ETO lacks the intranuclear targeting signal of the wild-type AML1 and is instead targeted by the ETO component to alternate nuclear matrix-associated sites leading to aberrant gene regulation and the leukemic disease. Several researchers have adopted strategies based on the AML1-ETO gene rearrangement for perturbating normal Runx transcriptional regulation in other systems. Specifically, a number of studies have used $\mathrm{COOH}$-terminal deletions of Runx2 as a dominant-negative tool for disrupting endogenous Runx2 activity in skeletal systems both in vivo and in vitro $(40,45-48)$. These proteins retain DNA binding but completely lack the $\mathrm{COOH}$ terminal sequences involved in coregulatory factor interactions and subnuclear targeting and, thus, are nonfunctional. In the present study, we use this approach to perturb the regulatory functions of the endogenous Runx 2 expressed in metastatic breast carcinoma to determine the role of Runx 2 in the generation of osteolytic disease.

\section{MATERIALS AND METHODS}

Generation of Stable Cell Lines. Stable cell lines were generated expressing the empty vector pcDNA 3.1 (Invitrogen Inc., San Diego, CA), full-length hemagglutinin-tagged mouse Runx2 or Xpress epitope-tagged deletion mutant (Runx2 $\Delta 230$ ) in the MDA-MB-231 or LCC15-MB human breast adenocarcinoma cell lines. The full-length hemagglutinin-tagged murine Runx2 or Xpress epitope-tagged deletion mutant (Runx2 $\Delta 230)$ are as described previously (40). Cells were transfected using LipofectAMINE (Invitrogen Inc.) according to the manufacturer's instructions and selected based on neomycin resistance (G418; Life Technologies, Inc./Invitrogen Corp., Grand Island, NY). Minimums of four independent clones were tested for each cell line construct combination.

Western Blot Analysis. Stable or untransfected cells were rinsed in PBS, trypsinized, and pelleted. Cell pellets were lysed in $300 \mu \mathrm{l}$ of lysis buffer [25 mm Tris-Cl (pH 7.5), $150 \mathrm{~mm} \mathrm{NaCl}, 1$ mm Na 2 EDTA, 1 mm EGTA, $1 \%$ Triton $\mathrm{X}-100,1 \% \mathrm{NP} 40,25 \mu \mathrm{M}$ MG132, and protease inhibitor mixture], and $50 \mu \mathrm{g}$ of protein were resolved on $10 \%$ SDS-PAGE. Blots were sequentially probed with monoclonal antibodies against Runx2 $(1: 3,000)$, Xpress epitope $(1: 5,000$; Santa Cruz Biotechnology, Santa Cruz, CA), and tubulin (1:25,000; Sigma Aldrich Inc.) with the blot stripped between each successive probing.

Surgical Protocols. Animal studies are conducted in accordance with approved Institutional Animal Care and Use Committee protocols and the NIH Guide for the Care and Use of Laboratory Animals. Tibial intramedullary injections were carried out on isofluorane anesthetized 4-6-week-old female severe combined immunodeficiency mice (The Jackson Laboratory, Bar Harbor, ME) using the technique described previously (49).

Preparation of Marrow Stromal Cells. Bone marrow stromal cell cultures were generated as described previously $(11,50,51)$. Five days after initial plating, 2500 (LCC15-MB) or 5000 (MDA-MB-231) human breast cancer cells were added to the cultures unless noted otherwise in text. Cultures were maintained with feeding every $48 \mathrm{~h}$ and till harvested at 21 days in $\alpha$-modified MEM containing $10 \%$ fetal bovine serum, Penn/Strep, $10^{-8} \mathrm{M}$ dexamethasone, $50 \mu \mathrm{g} / \mathrm{ml}$ ascorbic acid, and $8 \mathrm{~mm} \beta$-glycerol phosphate (all of the media and supplements were obtained from Life Technologies, Inc./Invitrogen Corp.).

Histology. Injected tibia were harvested and processed for paraffin embedding using the procedures described previously (51). Paraffin-embedded
5- $\mu \mathrm{m}$-thick sections were prepared and stained with $\mathrm{H} \& \mathrm{E}$ for a standard analysis of tissue sections. The adjacent sections were stained for tartrate resistant acid phosphatase (TRAP) activity as described previously (51).

RNase Protection Analysis (RPA). Total RNA was isolated from bone marrow stromal cell (BMSC) cocultures and control cultures using TRIzol (Invitrogen Corp.) as described previously (51). RPA was carried out using commercially produced template sets and reagents from PharMingen (BD Bioscience-PharMingen, San Diego, CA). The probe for TRAP was provided by Amgen, Inc. under a material transfer agreement. Band intensities were quantified and normalized using an $\alpha$ Inotech imaging system and associated chemiImager 4000 software ( $\alpha$ Inotech Corp., San Leandro, CA).

\section{RESULTS}

Characterization of Stable Cell Lines with Disrupted Runx2 Activity. In order to directly demonstrate a role for Runx2 in regulating tumor bone cell interactions, we generated a number of stably transfected breast cancer cell lines (both MDA-MB-231 and LCC15-MB based) that overexpressed the previously characterized COOH-terminal deleted mutant Runx 2 construct (Delta 230) or the full-length wild-type Runx2 depicted in Fig. 1A (40). Concurrently stable control lines were generated with the empty expression vector. We have reported previously that the Delta 230 construct is translocated to the nucleus and retains DNA binding affinity but does not support an independent transactivation activity for target genes (40). Studies from other laboratories demonstrate that $\mathrm{COOH}$-terminal deleted Runx2 protein functions by competing for DNA target site binding with the endogenous protein but displays
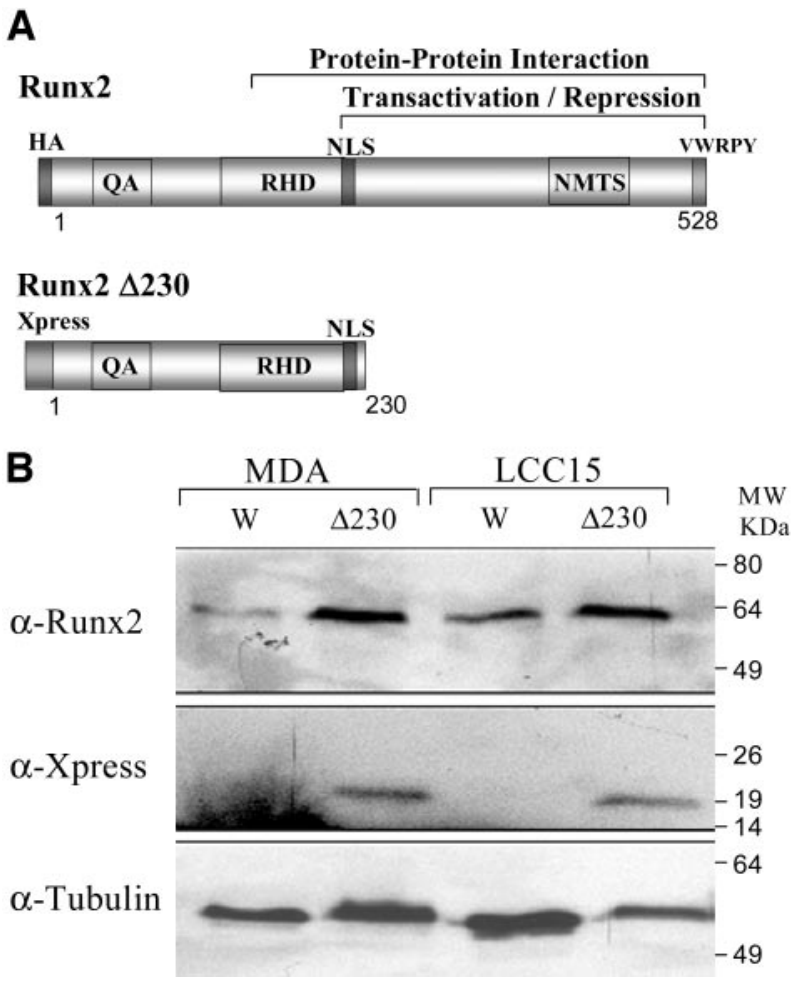

Fig. 1. A, diagrammatic representation of the mutant Runx2 (Runx2 $\Delta 230$ ) construct compared with the full-length wild-type protein (Runx2). Mutant construct is a deletion of the $\mathrm{COOH}$-terminal portion of the protein associated with the transactivation/repression activity. $B$, sequential Western blot analysis of endogenous ( $\alpha$-Runx2, top $)$ and the Xpress tagged mutant Runx2 protein ( $\alpha$-Xpress, middle). Stripped blots were then subsequently probed with $\alpha$-tubulin antibody to verify equal amounts of input protein. Runx 2 domains denoted by abbreviations in A: HA, hemagglutinin peptide tag; Xpress, Xpress peptide tag; $Q A$, polyglutamate-alanine rich region unique to Runx2; RHD, runt homology domain involved in target DNA binding and association with the $\mathrm{CBF} \beta$ cofactor; $N L S$, nuclear localization signal; NMTS, nuclear matrix targeting site; VWRPY, COOH-terminal sequence conserved in all of the members of the Runx family of factors. 


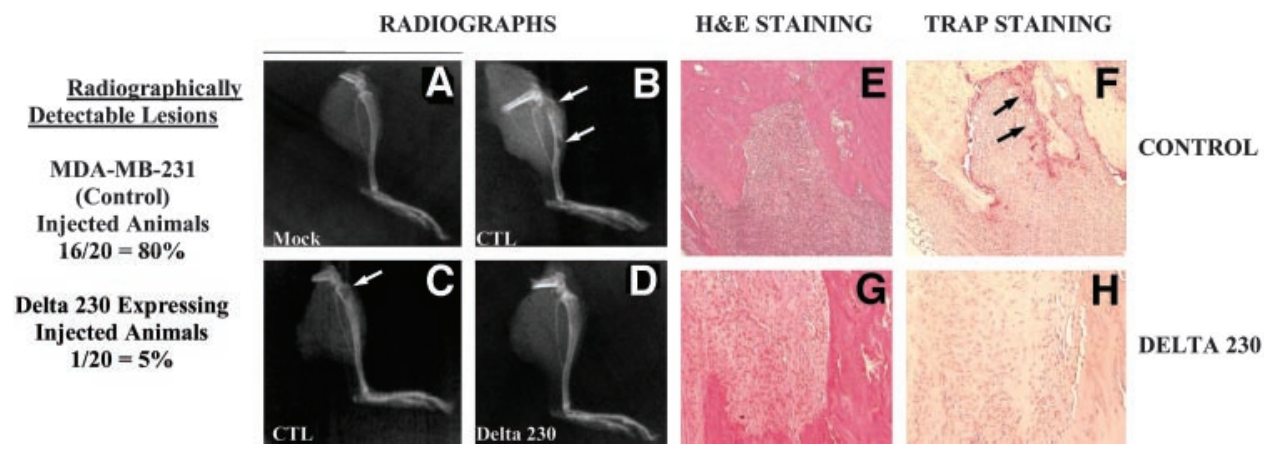

Fig. 2. Representative results of intramedullary injection experiments comparing control MDA-MB-231 to $\Delta 230$ mutant Runx expressing MDA-MB-231 cells. Radiographs of limbs

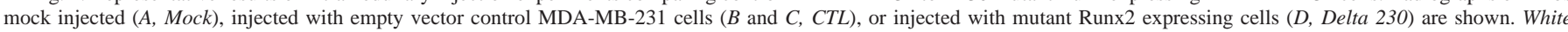

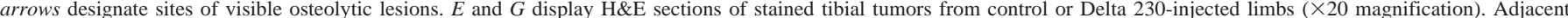

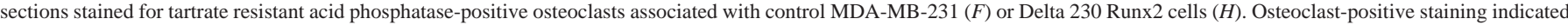
in $F$ by black arrows. TRAP, tartrate resistant acid phosphatase.

a higher DNA binding affinity and forms more stable protein DNA complexes than the wild-type molecules and, thus, acts as a potent competitive inhibitor (45). Functionally, these studies, as well as other in vivo studies, demonstrate that these mutant Runx2 proteins compete for and inhibit endogenous Runx 2 gene regulatory functions $(40,45-48)$.

For these studies, individual clonal cell lines were isolated after transfection of parental MDA-MB-231 or LCC15-MB breast adenocarcinoma cell lines with the mutant expression constructs and analyzed for transgene expression by Western blotting (representative blot displayed in Fig. $1 B$ ). On the basis of our previous studies demonstrating that Runx 2 acts as a positive regulator of BSP expression in human breast cancer cells, each cell line was additionally assessed for relative BSP promoter activity as a read-out for transgene inhibitory activity. Only cell lines that displayed a significant reduction in BSP promoter activity ( $>50 \%$ reduction) were selected for use in the subsequent studies (data not shown).

Disruption of Runx2 Activity in Breast Cancer Cells Abolishes the Formation of Osteolytic Lesions in Vivo. We initially compared the osteolytic activity of the MDA-MB-231 human breast cancer cell line engineered to stably overexpress the $\mathrm{COOH}$ terminal deleted Runx2, Delta 230, to the empty expression vectortransfected control line in an in vivo implantation model in immunocompromised severe combined immunodeficiency mice. In this model, cancer cells are injected directly into the intramedullary space of the tibia of a hind limb at a location where lytic lesions are commonly observed clinically in human cancers. Radiographic imaging of injected bones 8 weeks after implantation demonstrates that $80 \%$ (16 of 20) of control MDA-MB-231 cell-injected limbs display large, detectable osteolytic lesions (Fig. 2). In contrast, of the mice injected with the mutant Runx2 (Delta 230) expressing MDA-MB-231 cells, only 5\% (1 of 20) displayed radiographically detectable lesions. Histologically, the control line-associated lesions were large and surrounded by active osteoclasts as indicated by the extensive numbers of TRAP-positive staining cells along the tumor bone interface (Fig. 2F). The single lesion detected in mutant Runx2-injected limbs (1 of 20) was significantly smaller than controls and was not associated with TRAP-positive osteoclasts (Fig. 2H). These results demonstrate that the perturbation of Runx 2 regulatory function in breast cancer cells abolishes the ability of these cells to generate osteolytic lesions in bone.

Breast Cancer Cells Inhibit Osteoblast Differentiation in Vitro While Enhancing Osteoclast Differentiation. We next pursued a series of in vitro studies to define the response of bone marrowderived stromal cells to metastatic breast carcinoma cells. We compared two independent human breast cancer cell lines, MDAMB-231 and LCC15-MB, representing human breast adenocarcinoma of variable aggressiveness. The LCC15-MB, originally isolated from bone metastases in a breast cancer patient, is the more aggressive and displayed a higher relative level of Runx 2 protein (Fig. $1 B$ ). Five thousand cells/well of each breast cancer cell line was cocultured with $15 \times 10^{6}$ BMSC derived from C57BL/6J mice (11). Both human breast cancer cell lines inhibited osteoblastic differentiation of the BMSC as determined by counting the number of mineralized osteogenic colonies that formed (Fig. 3A). Individual nodules in these cultures represent an osteoblast colony that arises from the differentiation of an individual stem cell population (colony-forming unit), and, therefore, the nodule area represents the proliferation of that stem cell population (49). Coculture with tumor cells also reduced individual nodule areas by $5-10$-fold (Table 1). Osteogenic differentiation was additionally assessed biochemically by alkaline phosphatase activity and through the use of RPA of mature osteoblast-associated gene expression (BSP, osteocalcin, and collagen type I; Fig. $3, B$ and $C$, respectively). Coculture with the LCC15-MB cells nearly completely inhibited osteogenic differentiation of BMSC (nodules and alkaline phosphatase activity) and expression of bone phenotypic genes. All of the three parameters demonstrate an inhibition of mature osteoblast formation in cultures containing the breast cancer cells.

To demonstrate that our results were specific for the response of the murine marrow stromal cell population, we hybridized our human cellular RNAs to the murine species-specific RPA probes (Fig. 3D). In each template set used in these studies, the human RNAs did not hybridize to the specific murine-target gene probes; however, the human RNAs did hybridize with the internal control L32 probe producing an alternate-sized protected product. This result allows for an independent evaluation of human versus mouse cellular levels in coculture and shows that control and mutant Runx 2 expressing human breast cancer cells do not grow at significantly different rates (compare H.L32 to M.L32 bands in Fig. 5B, Lanes 2 and 3) in correlation with independent in vitro proliferation assays on these cell lines (data not shown).

We next examined the effect of coculture on the appearance of BMSC-derived osteoclasts. Our results demonstrate that in contrast to the inhibition of osteoblast differentiation, human breast cancer cells induce an increase in osteoclastic cell differentiation in BMSC. Our results show an overall increase in TRAP-positive staining cells and an increased number of multinucleated TRAP-positive staining cells (Fig. 4, $A-F$ ). Thus, the breast cancer cells increase TRAP + cell numbers $(\sim 2$-fold in cultures containing MDA-MB-231 and 3-fold 
A

Fig. 3. Human breast cancer cells mediate disruptions of normal murine bone marrow stromal cell osteoblast differentiation in an in vitro coculture. A, representative pictures of Von Kossa staining for osteoblast-associated mineralized nodule formation in cocultures. $B$, quantified osteoblastassociated alkaline phosphatase enzymatic activity. $C$, RNase protection analysis of murine osteoblastassociated gene expression in cocultures. Osteoblast genes include osteopontin $(O P N)$, bone sialoprotein $(B S P)$, collagen type I ( $\mathrm{Col} I)$, and osteocalcin $(O C)$. The housekeeping gene L32 probe hybridizes to both mouse and human RNAs (designated M.L32 and H.L32, respectively). D, RNase protection analysis of murine-specific osteoblast gene cassette cross-hybridization with isolated human RNAs (MDA = MDA-MB-231 an $\mathrm{LCC}=\mathrm{LCC} 15-\mathrm{MB}) . C$, lanes are designated as follows: $L$, undigested probe ladder; $M$, murine marrow stromal cells alone; 1, coculture with MDA-MB-231 cancer cells; and 2, coculture with LCC15-MB cancer cells. $D$, lanes are designated as follows: $L$, undigested ladder; $M D A$, MDA-MB231 cellular RNA; and $L C C$, LCC15-MB cellular RNA. bars, $\pm \mathrm{SD}$.

B
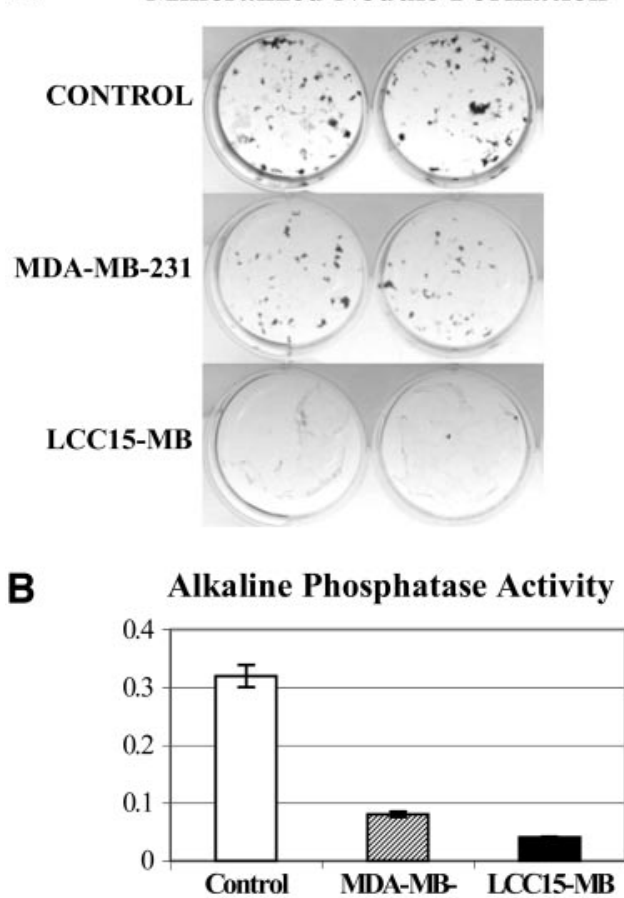

Osteoblast Gene Expression

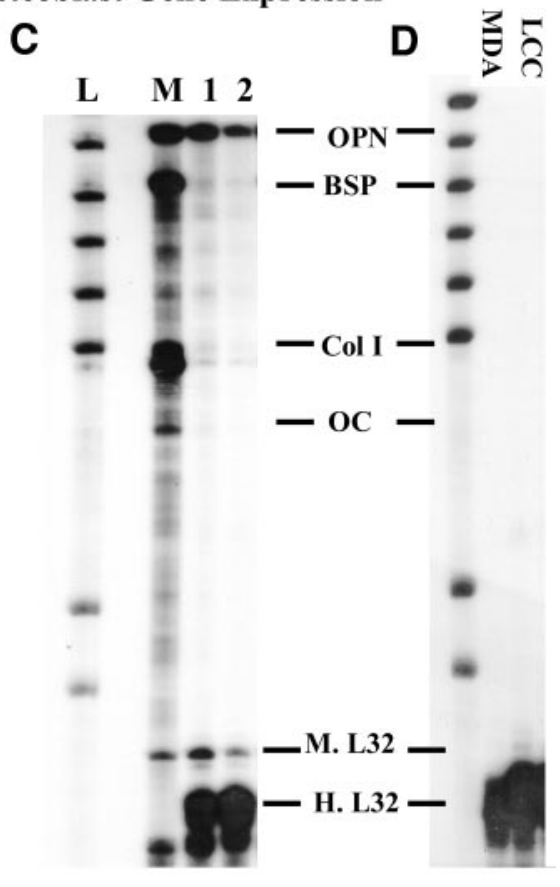

with LCC15-MB) but also osteoclast maturity as displayed by the increased number of multinucleated TRAP + staining cells. Molecular analyses using RPA demonstrate increased levels of TRAP gene expression, a marker of osteoclasts (Fig. 4G). Thus, breast cancer cells inhibit osteogenic differentiation while concurrently enhancing osteoclast differentiation. The inhibition of osteoblast differentiation combined with increased osteoclast differentiation is consistent with the conditions that would maximally produce a localized osteolytic bone loss.

Runx2 Regulates Breast Cancer Cell-Mediated Inhibition of Osteogenic Differentiation in Vitro. Additional coculture experiments demonstrate that Runx 2 regulatory function within the breast cancer cells is required for the tumor cell-associated inhibition of osteogenesis. Although control cancer cells inhibit osteoblast maturation, cocultures of BMSC with MDA-MB-231 (5000 cells/well) or LCC15-MB (2500 cells/well) that overexpress the Runx2, Delta 230 construct show comparable levels of mature osteoblasts to the control BMSC (Fig. 5A). Disruption of the endogenous Runx activity in these breast cancer cells rescued both the number of mineralized nodules formed and individual nodule areas showing that tumor cells inhibit both osteogenic stem cell differentiation and proliferation (Fig. 5A; Table 1). Using RNase protection assays, we demonstrate that disruption of Runx 2 activity in these cancer cell lines also rescues BMSC-associated expression of mature osteoblast marker genes, specifically bone BSP and osteocalcin, widely used markers of mature

Table 1 Quantified nodule data

\begin{tabular}{|c|c|c|c|}
\hline & Nodule number & Nodule area & Alkaline phosphatase \\
\hline \multirow{2}{*}{\multicolumn{4}{|c|}{$\begin{array}{l}\text { MDA-MB-231 } \\
\quad(5000 \text { cells/well })\end{array}$}} \\
\hline & & & \\
\hline MSC alone & $84^{(+/-6)}$ & $40237^{(+/-346)}$ & $0.42^{(+/-0.02)}$ \\
\hline Control & $27^{(+/-3)}$ & $6596^{(+/-62)}$ & $0.25^{(+/-0.01)}$ \\
\hline Delta 230 & $84^{(+/-7)}$ & $34607^{(+/-214)}$ & $0.42^{(+/-0.02)}$ \\
\hline \multicolumn{4}{|l|}{ LCC15-MB } \\
\hline (2500 cells/well) & & & \\
\hline MSC alone & $68^{(+/-4)}$ & $44508^{(+/-408)}$ & $0.45^{(+/-0.02)}$ \\
\hline Control & $21^{(+/-2)}$ & $4616^{(+/-23)}$ & $0.09^{(+/-0.01)}$ \\
\hline Delta 230 & $44^{(+1-3)}$ & $40883^{(+/-378)}$ & $0.40^{(+/-0.01)}$ \\
\hline
\end{tabular}

osteoblasts, in correlation with nodule formation (Fig. $5 B$, left). Thus, perturbation of Runx 2 transcriptional functions in breast cancer cells rescued osteoblast differentiation in cocultures.

In contrast, overexpression of Runx2 in MDA-MB-231 cells produces an increased inhibition in osteoblast differentiation as determined by Von Kossa staining of osteoblast-associated mineralized nodule formation (data not shown) and the decreased expression of osteoblast-associated genes (Fig. 5B, right).

Runx2 Regulates Breast Cancer Cell Induction of Osteoclast Formation and Marrow Stromal Cell Expression Cytokines That Regulate Osteoclastogenesis. Disruption of the endogenous Runx2 activity in human breast cancer cells also reduces osteoclast formation in cancer BMSC cocultures. Although cocultures containing control cancer cells displayed an increased expression of TRAP, overexpression of the mutant Runx construct in either the MDA-MB-231 or LCC15-MB cell line abrogated this osteoclast differentiation effect (Fig. 6, $A$ and $B$ ). An analysis of murine stromal cell expression of proresorptive cytokines in cocultures demonstrates that tumor cells elicited an increase in the BMSCassociated expression of multiple cytokines known to influence osteoclast differentiation (Fig. 7). Specifically, our results show that breast cancer cells enhanced BMSC expression of inducible nitrous oxide, tumor necrosis factor $\alpha$, RANKL, and IL-6, each of which is known to enhance osteoclast differentiation, activity, and/or survival $(8,11-14)$. RANKL, specifically, is one of the primary inductive factors of new osteoclast differentiation and has been associated with the generation of osteolysis (13). Disruption of Runx 2 activity with the mutant Runx construct abolished the increased expression of these osteoclastogenic cytokines demonstrating that Runx2 is a regulator of the influence of the cancer cell on multiple factors within the bone marrow microenvironment that affect osteoclast differentiation (Fig. 7).

\section{DISCUSSION}

The osteolytic disease associated with metastases of carcinoma in bone is the consequence of two related events. The first is the 


\section{Histochemical TRAP Staining}

Control MSC

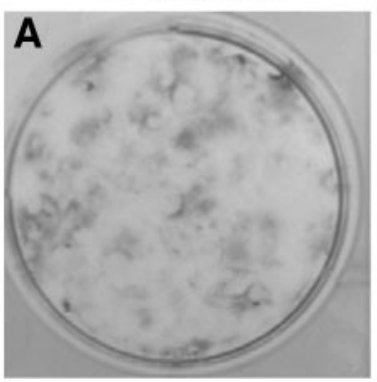

MSC + MDA cells
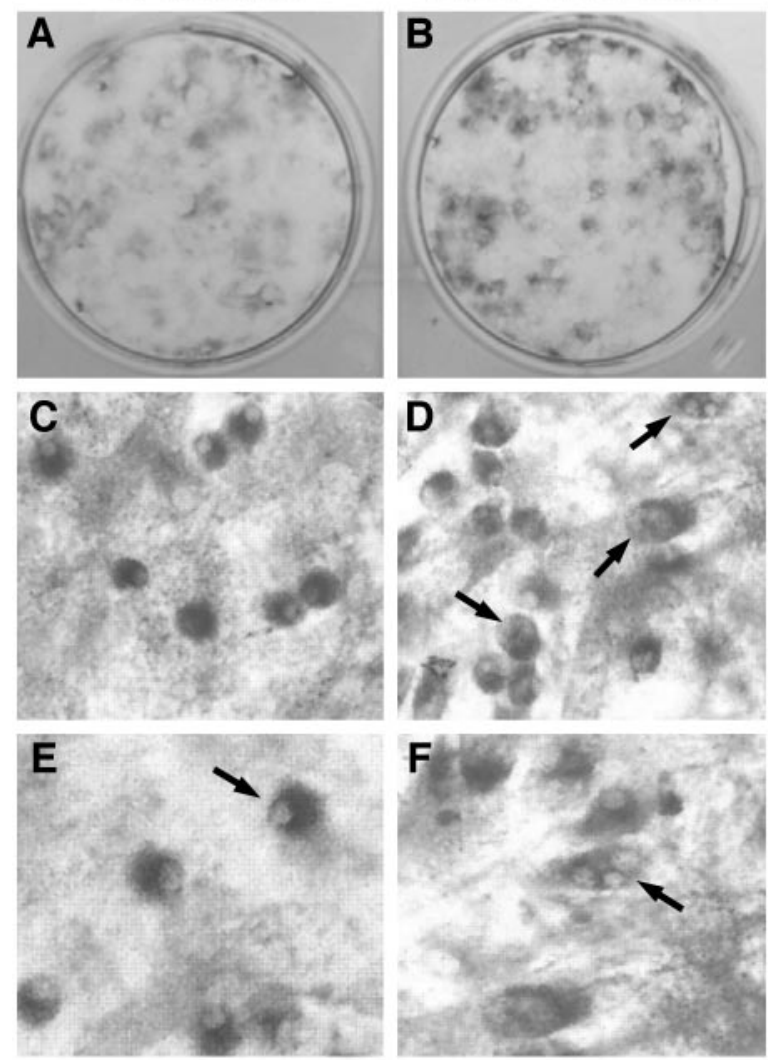

G Normalized TRAP Gene Expression

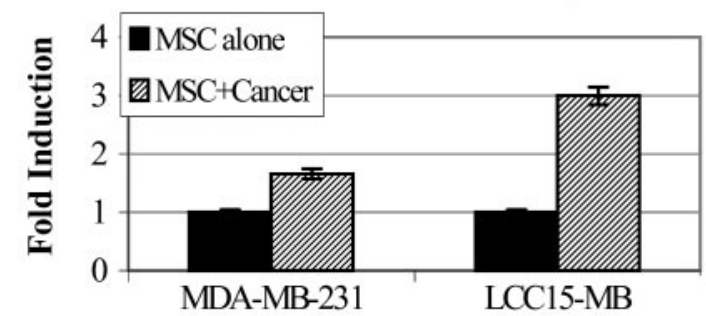

Fig. 4. Human breast cancer cells mediate disruptions of normal murine bone marrow stromal cell osteoclast differentiation in an in vitro coculture. $A-F$, representative photomicrographs of histochemical staining for osteoclast lineage-associated cellular tartrate resistant acid phosphatase (TRAP) activity in bone marrow stromal cell control versus MDA-MB-231 cancer cell cocultures. $A$ and $B$, low magnification pictures of individual well of six-well plate. $C$ and $D, \times 20$ magnification of individual cellular staining in a representative field. Note control cultures contain predominantly TRAP-positive cells with single nuclei as compared with cocultures that contain multinucleated $(3+)$ as indicated by arrows. E, $\times 40$ magnification of single nucleated TRAP-positive cell in control bone marrow stromal cell cultures (arrow). $F, \times 40$ magnification of multinucleated (3+) TRAP-positive cells in coculture with MDA-MB-231 cancer cells (arrows). $G$ graphical representation of normalized TRAP expression. bars, $\pm \mathrm{SD}$.

ability of the tumor cells to seed and grow in the ectopic environment. This requires that the tumor cells be capable of responding to the local morphogens in the new environment. The second component is the ability of tumor cells to produce factors that modify the local environment, thus, additionally enhancing the growth of these tumors. This is commonly referred to as the "viscous cycle" and represents the efforts of the cancer to mobilize increasing amounts of morphogens by increasing bone removal and the release of the factors sequestered in the bone matrix $(2,8$, 11-14). Thus, the osteolytic bone destruction associated with me- tastases is not an aberrant influence of cancer cells on bone, but rather the purposeful mobilization of morphogens sequestered in bone for the benefit of tumor cells.

In the studies presented here, we have used both in vivo and in vitro models to functionally test the importance of Runx 2 in regulating the interactions between breast cancer cells and the bone microenvironment. In vivo, we have used a model of direct injection of human breast cancer cells in the marrow cavity (intramedullary space) of the tibial bone. This model allows for the growth and disease associated with the cancer cells to occur in the specific environment they find after metastasis. Our results demonstrate that perturbation of Runx2 regulatory function in breast cancer cells abolishes their ability to form osteolytic lesions in bone (Fig. 2). We observe that cells expressing mutant Runx 2 do not form tumors when injected, independ-

A

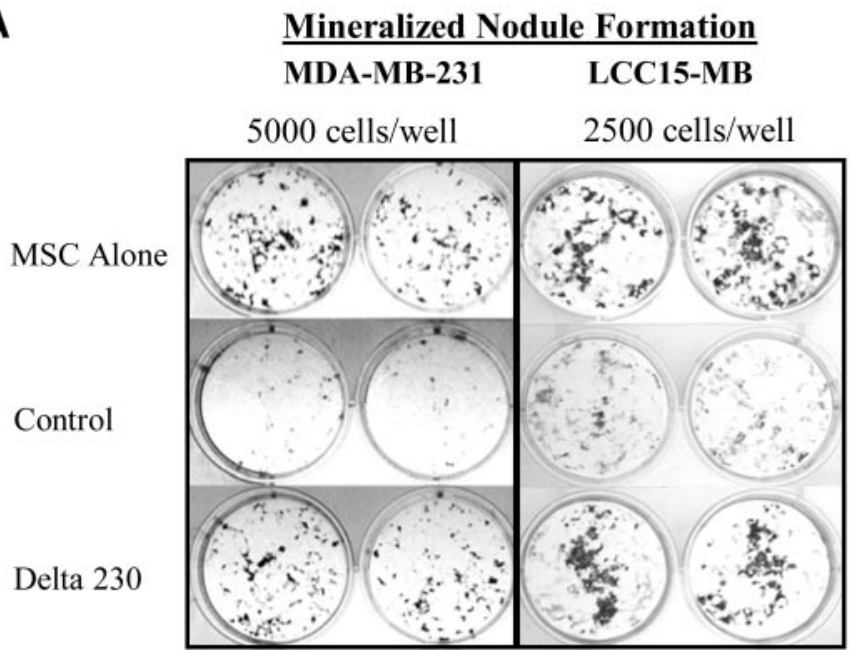

B

Osteoblast Gene Expression
Disruption of Runx2

MDA-MB-23

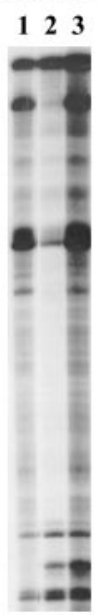

LCC15-MB

123

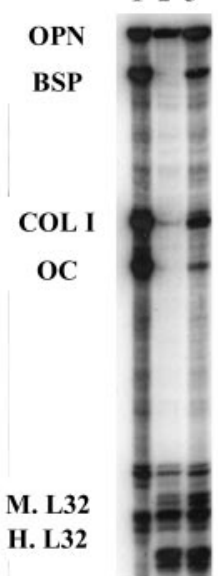

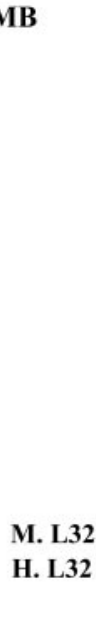

Over-expression of Runx2

\author{
MDA-MB-231
}

$\begin{array}{llll}1 & 2 & 4\end{array}$

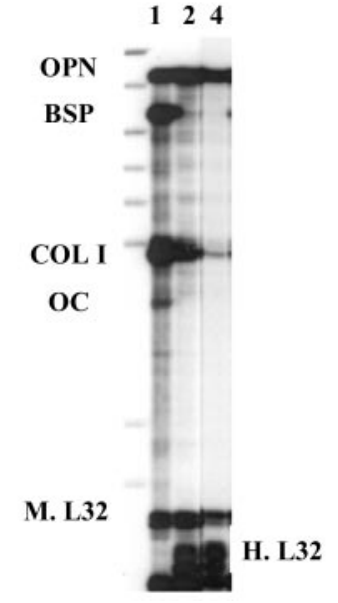

Fig. 5. Cancer cell-mediated disruptions of osteoblast differentiation require Runx2 activity in breast cancer cells. A, representative picture of Von Kossa staining of osteoblast-associated mineralized nodule formation in marrow stromal cell cocultures alone (MSC alone) or with cancer cells expressing either empty vector (control) or the mutant Runx 2 construct as indicated in the panel. $B$, RNase protection analysis of osteoblastassociated gene expression in marrow stromal cells cultured alone $(B$, Lane 1$)$, coculture with control cancer cells, MDA-MB-231 or LCC15-MB as designated in the figure (Lane 2), coculture with Delta 230 Runx2 expressing MDA-MB-231 or LCC15-MB cancer cells (Lane 3), or MDA-MB-231 cancer cells overexpressing Runx2 (Lane 4, right). Osteoblast-associated genes include osteopontin $(O P N)$, bone sialoprotein $(B S P)$, collagen type I (Col I), and osteocalcin (OC). Murine L32 (M.L32) and human L32 (H.L32) bands are indicated on the figure. 
A

TRAP Gene Expression

MDA-MB-231 LCC15-MB

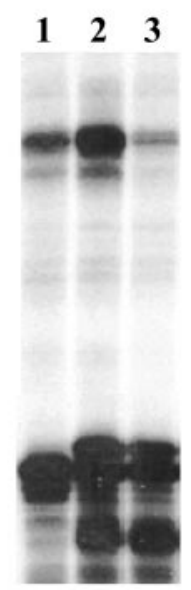

$\begin{array}{lll}1 & 2 & 3\end{array}$
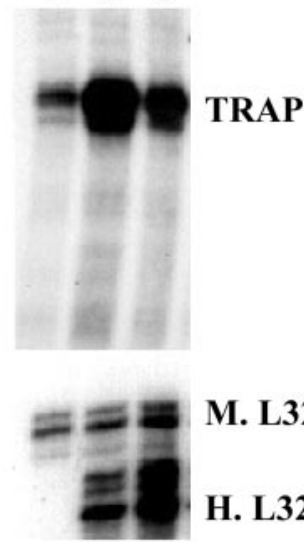

M. L32

H. L32

\section{B}

Normalized TRAP Gene Expression

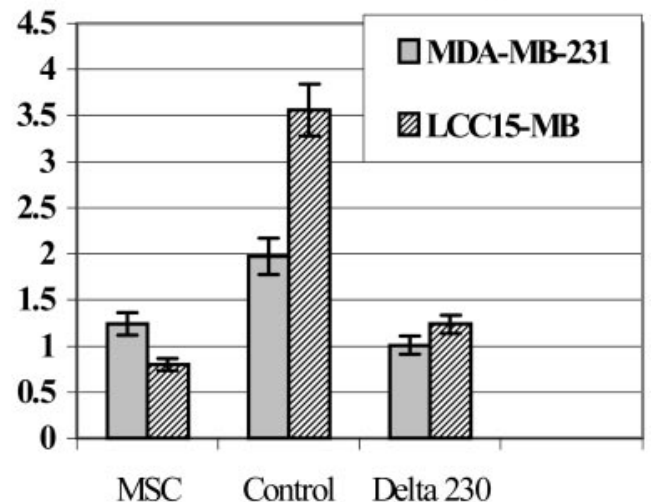

Fig. 6. Enhancement of osteoclast differentiation in bone marrow stromal cell coculture requires Runx 2 activity in breast cancer cells. $A$, RNase protection analysis of osteoclastassociated tartrate resistant acid phosphatase (TRAP) expression. $B$, quantification of normalized RNase protection analysis results from $A$. Lanes are designated as follows in $A$ : 1 , marrow stromal cells alone; 2 , coculture with control cancer cells; and 3, coculture with cancer cells expressing the Delta 230 Runx2. Murine L32 (M.L32) and human L32 (H.L32) controls are as indicated. bars, \pm SD.

ent of the appearance of osteolytic lesions, suggesting that disruption of Runx 2 impacts the breast cancer cell growth in bone. We also observed that the lesion formed in limbs injected with breast cancer cells expressing mutant is not associated with active osteoclasts. This observation shows that independent of growth, tumor cells lacking intact Runx2 function do not activate local osteoclasts. This is consistent with our in vitro data (Fig. 6). Thus, our results support the conclusion that Runx 2 activity is required for both the response of tumor cells to the bone environment and the influence of tumor cells on bone cell differentiation.

The second model used in these studies is an in vitro coculture system of breast cancer cells mixed with bone marrow-derived stromal cells representing the cellular compartment of the bone. These cocultures allow for the independent analysis of the responses of both the bone marrow stromal elements and the human cancer cells through the use of species-specific genetic probes. In our current studies, we focus on the response of local bone cells through the use of species-specific probes and analysis of specific genes associated with the osteoblast and osteoclast differentiation. Our experimental results demonstrate that human breast cancer cells inhibit osteoblast maturation in these cocultures while con- currently enhancing osteoclast formation. In addition, we demonstrate that tumor cells elicit an increase in the BMSC expression of cytokine inducers of osteoclast formation and activity. It has been reported previously that cancer cells induce enhanced expression of RANKL in osteoblasts in response to either IL-8 or parathyroid hormone-releasing protein and that this may be the result of a switch from the bone forming to the osteoclast coupling functions in osteoblasts $(7,8,11-14)$. Our data support this conclusion and additionally suggest that the coupling function of osteoblasts is primarily carried out by immature osteoblasts given the observed inhibition of osteoblast maturation concomitant with the increased expression of inductive cytokines. This conclusion is supported by

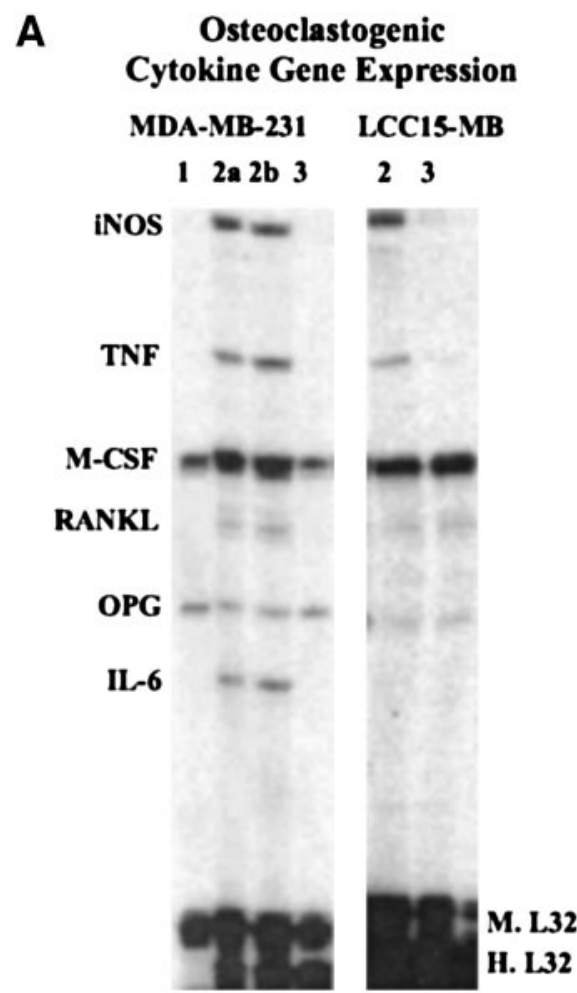

\section{B Quantified Cytokine Expression Data}

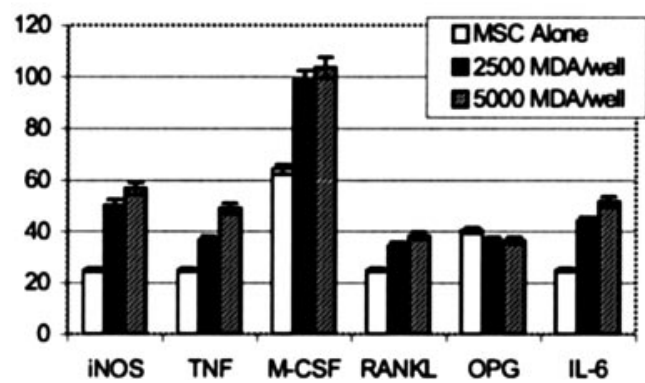

Fig. 7. Human breast cancer cell induction of increased pro-osteoclastogenic cytokine expression in murine marrow stromal cells requires Runx2. A, RNase protection analysis of murine cytokine expression in bone marrow stromal cells. Osteoclastogenic cytokines include inducible nitrous oxide (iNOS), tumor necrosis factor $\alpha(T N F)$, macrophage colony stimulating factor $(M-C S F)$, receptor activator of nuclear factor- $\kappa \mathrm{B}$ ligand $(R A N K L)$, osteoprotogerin $(O P G)$, and interleukin $6(I L-6)$. $B$, graphical presentation of normalized RNase protection data comparing control bone marrow stromal cells to cocultures containing either 2500 or 5000 MDA-MB231 cells/well. A, lanes are designated as follows: 1, marrow stromal cells alone; 2 , coculture with control cancer cells; and 3, coculture with cancer cells expressing the mutant Runx2. Lanes $2 a$ and $2 b$ represent coculture with the control MDA-MB-231 cancer cells added at either 2500 cells/well $(2 a)$ or 5000 cells/well $(2 b)$. LCC15-MB cancer cells are added at 2500 cells/well. Murine L32 (M.L32) and human L32 (H.L32) controls are as indicated. bars, \pm SD. 
the recent report demonstrating that RANKL is produced primarily by immature osteoblasts (52).

Perturbation of Runx 2 function in the human breast cancer cells demonstrates that Runx2 regulates the influence of cancer cells on the local bone cellular compartment. Overexpression of the mutant Runx 2 construct in either the MDA-MB-231 or LCC15-MB cells abolished the tumor cell-associated inhibition of osteoblast maturation (Fig. 5; Table 1), enhanced osteoclast formation, and the increased BMSC expression of cytokines that induce osteoclast formation and activity (Figs. 6 and 7, respectively). Although our results do not discriminate between direct and indirect influences of tumor cells on osteoclast differentiation, these results are consistent with the model proposed previously in which the primary influence of metastatic breast carcinoma cells is on the osteoblast (8, 11-14). Our data specifically demonstrate that the primary influence of breast cancer cells on osteoblasts is an inhibition of their maturation. The inhibition of osteoblast differentiation and associated new bone formation alone would lead eventually to localized bone loss. Such an inhibition would also generate an increase in the local number of immature osteoblasts and, thus, explain the observed associated increase in the expression of the cytokines that induce osteoclast formation expressed by these cells. An increase in the number of osteoclasts would be favored under these conditions leading to an increased rate of bone removal and osteolytic destruction of local bone. Thus, our results suggest breast cancer cells generate osteolytic disease by concurrently inhibiting new bone formation and enhancing localized bone removal. Our experimental results demonstrate that fidelity of Runx2 function in human metastatic breast cancer cells is required for tumor cells to elicit both of these effects on bone.

\section{ACKNOWLEDGMENTS}

We thank Cory Edgar and Jennifer Fitch for technical assistance during the course of these experiments and Dr. Andre Van Wijnen for critical reading of the manuscript.

\section{REFERENCES}

1. Paget S. The distribution of secondary growths in cancer of the breast. Lancet $1889 ; 1: 571-3$.

2. Mundy GR. Mechanisms of bone metastasis. Cancer (Phila) 1997;80:1572-80.

3. Boyde A, Maconnachie E, Reid SA, Delling G, Mundy GR. Scanning electron microscopy in bone pathology: review of methods. Potential and implications. Scanning Electron Microsc 1986;IV:1537-54.

4. Diel IJ, Slomayer EF, Costa SD, et al. Reduction in new metastases in breast cancer with adjuvant clodronate treatment. N Engl J Med 1998;339:357-63.

5. Hortobagyi GN, Theriault RL, Porter L, et al. Efficacy of pamidronate in reducing skeletal complications in patients with breast cancer and lytic bone metastases. Protocol 19 Aredia Breast Cancer Study Group. N Engl J Med 1996;335: $1785-91$.

6. Bendre MS, Gaddy-Kurten D, Mon-Foote T, et al. Expression of interleukin 8 and not parathyroid hormone-related protein by human breast cancer cells correlates with bone metastasis in vivo. Cancer Res 2002;62:5571-79.

7. Bendre MS, Montague DC, Peery T, et al. Interleukin- 8 stimulation of osteoclastogenesis and bone resorption is a mechanism for the increased osteolysis of metastatic bone disease. Bone 2003;33:28-37.

8. Mancino AT, Klimberg VS, Yamamoto M, Manolagas SC, Abe E. Breast cancer increases osteoclastogenesis by secreting M-CSF and upregulating RANKL in stromal cells. J Surg Res 2001;100:18-24.

9. Sotiriou C, Lacroix M, Lespagnard L, et al. Interleukins-6 and-11 expression in primary breast cancer and subsequent development of bone metastases. Cancer Lett 2001;169:87-95.

10. Suarez-Cuervo C, Harris KW, Kallman L, Vaananen HK, Selander KS. Tumor necrosis factor-alpha induces interleukin-6 production via extracellular-regulated kinase 1 activation in breast cancer. Breast Cancer Res Treat 2003;80:71-8

11. Thomas RJ, Guise TA, Yin JJ, et al. Breast cancer cells interact with osteoblasts to support osteoclast formation. Endocrinology 1999;140:4451-8.

12. Chikatsu N, Takeuchi $\mathrm{Y}$, Tamura $\mathrm{Y}$, et al. Interactions between cancer and bone marrow cells induce osteoclast differentiation factor expression and osteoclast-like cell formation in vitro. Biochem Biophys Res Commun 2000;267:632-7.
13. Kitazawa S, Kitazawa R. RANK ligand is a prerequisite for cancer-associated osteolytic lesions. J Pathol 2002;198:228-36.

14. Kakonen S-M, Mundy GR. Mechanisms of osteolytic bone metastases in breast carcinoma. Cancer (Phila) 2002;97:834-9.

15. Bellahcene A, Kroll M, Liebens F, Castronovo V. Bone sialoprotein expression in primary human breast cancer is associated with bone metastases development. J Bone Miner Res 1996;11:665-70.

16. Waltregny D, Bellahcene A, de Leval X, et al. Increased expression of bone sialoprotein in bone metastases compared with visceral metastases in human breast and prostate cancers. J Bone Miner Res 2000;15:834-43.

17. Barnes GL, Javed A, Kamal MH, et al. Osteoblast related transcription factors Runx2 (Cbfa1/AML3) and MSX2 mediate the expression of bone sialoprotein in human metastatic breast cancer cells. Cancer Res 2003;63:2631-7.

18. Selvamurugen N, Partridge NC. Constitutive expression and regulation of collagenase-3 in human breast cancer cells. Mol Cell Biol Res Commun 2000;3:218-23.

19. Inman CK, Shore P. The osteoblast transcription factor Runx 2 is expressed in mammary epithelial cells and mediates osteopontin expression. J Biol Chem 2003; 278:48684-9.

20. Komori T, Kishimoto T. Cbfa1 in bone development. Curr Opin Genet Dev 1998;8 494-9.

21. Lian JB, Stein GS. Runx2/Cbfa1: a multifunctional regulator of bone formation. Curr Pharm Des 2003;9:2677-85

22. Ducy P, Zhang R, Geoffrey V, Ridall AL, Karsenty G. Osf2/Cbfa1: a transcriptional activator of osteoblast differentiation. Cell 1997;89:677-80.

23. Komori T, Yagi H, Nomura S, et al. Targeted disruption of Cbfa1 results in a complete lack of bone formation owing to maturational arrest of osteoblasts. Cell 1997;89:755-64.

24. Banerjee C, McCabe LR, Choi JY, et al. Runt homology domain proteins in osteoblast differentiation: AML3/Cbfa1 is a major component of a bone specific complex. J Cell Biochem 1997;66:1-8.

25. Pratap J, Galindo M, Zaidi SK, et al. Cell growth regulatory role of Runx2 during proliferative expansion of preosteoblasts. Cancer Res 2003;63:5357-62.

26. Thirunavukkarasu K, Miles RR, Halladay DL, et al. The osteoblast-specific transcription factor Cbfal contributes to the expression of osteoprotogerin, a potent inhibitor of osteoclast differentiation and function. J Biol Chem 2000;275:25163-72.

27. Gao YH, Shinki T, Yuasa T, et al. Potential role of Cbfa1, an essential transcriptional factor for osteoblast differentiation, in osteoclastogenesis: regulation of mRNA expression of osteoclast differentiation factor (ODF). Biochem Biophys Res Commun 1998;252:697-702.

28. Enomoto H, Shiojiri S, Hoshi K, et al. Induction of osteoclast differentiation by Runx2 through receptor activator of nuclear factor-kappa B ligand (RANKL) and osteoprotogerin regulation and partial rescue of osteoclastogenesis in Runx2-1mice by RANKL transgene. J Biol Chem 2003;278:23971-7.

29. Zelzer E, Glotzer DJ, Hartmann C, et al. Tissue specific regulation of VEGF expression during bone development requires Cbfa1/Runx2. Mech Dev 2001;106: 97-106.

30. Porte D, Tuckerman J, Becker M, et al. Both AP-1 and Cbfa1-like factors are required for the induction of interstitial collagenase by parathyroid hormone. Oncogene 1999; 18:667-78.

31. Jimenez MJ, Balbin M, Lopez JM, et al. Collagenase 3 is a target of Cbfa1, a transcription factor of the runt gene family involved in bone formation. Mol Cell Biol 1999; 19:4431-42.

32. Harada H, Tagashira S, Fujiwara M, et al. Cbfa1 isoforms exert functional differences in osteoblast differentiation. J Biol Chem 1999;274:6972-8.

33. Javed A, Barnes GL, Jasanya BO, et al. Runt homology domain transcription factors (Runx, Cbfa, and AML) mediate repression of the bone sialoprotein promoter: evidence for promoter context-dependent activity of Cbfa proteins. Mol Cell Biol 2001;21:2891-905.

34. Stein GS, Lian JB, Stein JL, et al. Intranuclear organization of Runx transcriptional regulatory machinery in biological control of skeletogenesis and cancer. Blood Cells Mol Dis 2003;30:170-6.

35. Aronson BD, Fisher AL, Blechman K, Caudy M, Gergen JP. Groucho-dependent and independent repression activities of Runt domain proteins. Mol Cell Biol 1997; 17: 5581-7.

36. Hanai J, Chen LF, Kanno T, et al. Interaction and functional cooperation of PEBP2/ CBF with smads. Synergistic induction of the immunoglobulin germline ca promoter. J Biol Chem 1999;274:31577-82.

37. Javed A, Guo B, Hiebert S, et al. Groucho/TLE/R-Esp proteins associate with the nuclear matrix and repress Runx dependent activation of tissue-specific gene transcription. J Cell Sci 2000;113:2221-31.

38. Yagi R, Chen LF, Shigesada K, Murakami Y, Ito Y. A WW domain-containing Yes-associated protein (YAP) is a novel transcriptional co-activator. EMBO J 1999; 18:2551-62.

39. Zhang YW, Yasui N, Kakazu N, et al. A Runx2/PEBP2alphaA/Cbfa1 mutation displaying impaired transactivation and smad interaction in cleidocranial dysplasia. Proc Natl Acad Sci USA 2000;97:10549-54.

40. Gutierrez S, Javed A, Tennant DK, et al. CCAAT/enhancer-binding proteins (C/EBP) $\beta$ and $\delta$ activate osteocalcin gene transcription and synergize with Runx2 at the C/EBP element to regulate bone-specific expression. J Biol Chem 2002;277: 1316-23.

41. Stein GS, Lian JB, Stein JL, et al. Temporal and spatial parameters of skeletal gene expression: targeting Runx factors and their co-regulatory proteins to subnuclear domains. Connect Tissue Res 2003;44:149-53.

42. Lian JB, Stein JL, Stein GS, et al. Runx2/Cbfa1 functions: diverse regulation of gene transcription by chromatin remodeling and co-regulatory protein interactions. Connect Tissue Res 2003;44:141-8. 
43. Barseguian K, Lutterbach B, Hiebert SW, et al. Multiple subnuclear targeting signals of the leukemia-related AML1/ETO and ETO repressor proteins. Proc Natl Acad Sci USA 2002;99:15434-9.

44. Downing JR. The aml-1-eto chimeric transcription factor in acute myeloid leukemia: biology and clinical significance. Br J of Haematol 1999;106:296-308.

45. Ducy P, Starbuck M, Priemel M, et al. A Cbfa1-dependent genetic pathway controls bone formation beyond embryonic development. Genes Dev 1999;13: $1025-36$.

46. Ueta C, Iwamoto M, Kanatani N, et al. Skeletal malformations caused by over expression of Cbfal or its dominant negative form in chondrocytes. J Cell Biol 2001;153:87-99.

47. Iwamoto M, Kitagaki J, Tamamura Y, et al. Runx2 expression and action in chondrocytes are regulated by retinoid signaling and parathyroid hormone-related peptide (PTHrp). Osteoarthritis Cartilage 2003;11:6-15.
48. Sun L, Vitolo M, Passaniti A. Runt-related gene 2 in endothelial cells: inducible expression and specific regulation of cell migration and invasion. Cancer Res 2001; 61:4994-5001.

49. Lee Y, Schwarz E, Davies M, et al. Differences in cytokine profiles associated with prostate cancer cell induced osteoblastic and osteolytic lesions in bone. J Orthop Res 2003;21:62-72.

50. Kuznetsov SA, Kresbach PM, Satomura K, et al. Single-colony derived strains of human marrow stromal fibroblasts form bone after transplantation in vivo. J Bone Miner Res 1997;12:1335-47.

51. Gerstenfeld LC, Cho TJ, Kon T, et al. Impaired fracture healing in the absence of TNF-a signaling: the role of TNF-a in endochondral cartilage resorption. J Bone Miner Res 2003;18:1584-92.

52. Atkins GJ, Kostakis P, Pan B, et al. RANKL expression is related to the differentiation state of human osteoblasts. J Bone Miner Res 2003;18:1088-98. 